\title{
The Lyman- $\alpha$ forest as a probe of fundamental physics
}

\author{
Matteo Viel $^{1}$ \\ ${ }^{1}$ Institute of Astronomy, University of Cambridge, Cambridge, CB3 0HA, UK \\ email: viel@ast.cam.ac.uk
}

\begin{abstract}
We use LUQAS, a sample of 27 high resolution high signal-to-noise UVES quasar (QSO) spectra (Kim et al. 2004), and the Croft et al. (2002) sample together with a set of high resolution large box-size hydrodynamical simulations run with the code GADGET-II to recover the linear dark matter power spectrum at $z>2$ and at scales of $1-40 h^{-1} \mathrm{Mpc}$. These scales cannot be probed by any other observable. We address some of the uncertainties in the theoretical modelling of the Lyman- $\alpha$ forest structures such as the difference between full hydro-dynamical simulations and a more simplified scheme based on the HPM (Hydro-ParticleMesh) technique. We combine these data sets with the Cosmic Microwave Background (CMB) data of WMAP in order to get tighter constraints on cosmological parameters. We focus on the recovered values of the power spectrum amplitude, the primordial spectral index and the running of the primordial spectral index. By considering models of slow-roll inflation we give constraints on inflationary parameters. We explore the implications for the mass of a warm dark matter particle, including light gravitinos and sterile neutrinos, and from the perspective of constraining the neutrino mass.
\end{abstract}

\section{Introduction}

The prominent absorption features blue-ward of the Lyman- $\alpha$ emission in the spectra of high-redshift quasars (QSOs) are now generally believed to arise from smooth density fluctuations of a photoionised warm intergalactic medium (e.g. Rauch 1998). This has opened up the possibility to probe the density fluctuations of matter with the flux power spectrum of QSO absorption lines (Croft et al. 1998; McDonald et al. 2000; Gnedin \& Hamilton 2001; Hui et al. 2001; Croft et al. 2002; McDonald et al. 2004; Viel, Haehnelt \& Springel 2004). The flux power spectrum is mainly sensitive to the slope and amplitude of the linear dark matter power spectrum for wave-numbers in the range $0.002<k$ $(\mathrm{s} / \mathrm{km})<0.05$, roughly corresponding to scales $1-40$ co-moving Mpc/h. We use a suite of high-resolution hydrodynamical simulations and the flux power spectrum obtained from LUQAS (Large sample of UVES QSO Absorption Spectra), together with the published flux power spectrum of Croft et al. (2002), to infer the linear dark matter power spectrum at $z=2.125$ and $z=2.72$. The idea is to rely on accurate high resolution and large boxsize hydrodynamical simulations to model the bias function $b(k)$ which relates the flux to the linear dark matter power spectrum: $P_{F}(k)=b^{2}(k) P(k)$. Moreover, we will briefly address some of the uncertainties that arise from theoretical (numerical) modelling of the Lyman- $\alpha$ forest structures.

We will then combine these estimates, in the framework of Monte Carlo Markov Chains (Lewis \& Bridle 2002), with the Cosmic Microwave Background (CMB) results of WMAP (Spergel et al. 2003) to investigate the following issues: i) recovery of cosmological parameters such as $\sigma_{8}$ (the power spectrum amplitude), $n_{s}$, and $n_{\text {run }}$ (the spectral index and its running); ii) constraints on parameters describing slow-roll inflationary models; and iii) constraints on dark matter particle masses and neutrinos. 

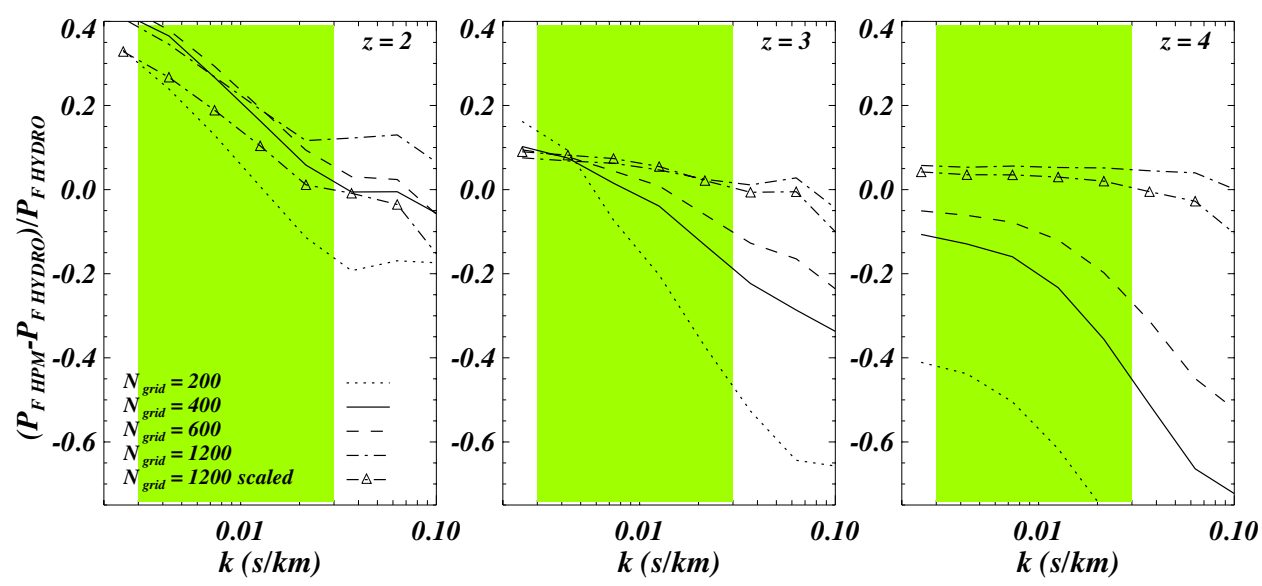

Figure 1. Fractional differences between the flux power spectra computed from full hydrodynamical simulations and HPM simulations with different resolutions for the grid size. The shaded area indicates the wavenumber range used for the recovery of the underlying linear dark matter power spectrum.

\section{The LUQAS and the Croft et al. (2002) samples}

The LUQAS sample consists of 27 QSO spectra taken with the Ultra-Violet Echelle Spectrograph (UVES) on VLT. Most of the spectra have been taken as part of the Large ESO Observing programme UVESLP (P.I.: J. Bergeron). The median redshift of the sample is $z=2.125$ and the total redshift path is $\Delta z=13.75$. The typical signal-to-noise ratio is $\sim 50$ and the pixel size is $0.05 \AA$. For a more detailed description of the sample and the data reduction we refer to Kim et al. (2004). We combine this sample with that of Croft et al. (2002), which consists of 30 Keck HIRES spectra and 23 Keck LRIS spectra and has a median redshift of $z=2.72$. These two data sets seem to be in agreement with the flux power spectrum obtained by McDonald et al. (2004) and Viel et al. (2004) from a large sample of low-resolution SDSS spectra. Although some differences between the different data sets are present, at the level of the error bars that we are going to quote, these discrepancies are not relevant. In all the data sets the systematic uncertainties are larger than the statistical errors (Kim et al. 2004; McDonald et al. 2004; Viel, Haehnelt \& Springel 2004).

\section{Hydrodynamical simulations: HPM vs. full hydro}

Presently, one of the biggest sources of error is due to the not very well understood numerical modelling of the Lyman- $\alpha$ forest (Viel, Haehnelt \& Springel 2004). The HydroParticle-Mesh (HPM) method is an approximate technique to simulate the low density IGM (Gnedin \& Hui 1998) and has been extensively used to recover cosmological parameters from Lyman- $\alpha$ forest data (McDonald et al. 2004; Seljak et al. 2004). This method is based on the numerical implementation of an effective potential, that mimics gas pressure, into a particle mesh code that simulates collisionless dark matter. We decided to test the accuracy of this method in reproducing the flux power spectrum, which is the quantity that is used to infer the dark matter power spectrum, using state-of-the-art hydrodynamical simulations run with the GADGET-II code. The results are shown in Fig. 1 where we plot the fractional differences between the flux power spectrum of HPM simulations and the full hydrodynamical one. We can see that if the resolution $\left(N_{\text {grid }}\right)$ 
of the HPM method increases, then the differences can be as small as $5-10 \%$ at $z=3,4$ (middle and left panels), in the wavenumber range of interest in the analysis (represented by the shaded area). At $z=2$ there are strong discrepancies even at large scales that are due to the large amount of shock-heated gas present in the full hydrodynamical simulations that cannot be properly modelled with the HPM technique. The line with over-plotted empty triangles represents the case in which the spectra have been scaled to match the same mean flux (both in the HPM and full hydro runs), a procedure which is usually adopted by simulators. We note that this scaling determines a better but still not perfect agreement of the two flux power spectra. Caution is thereby needed when using HPM simulations to infer cosmological parameters, and we are compelled to accurately calibrate these simulations with full hydrodynamical ones (Viel, Haehnelt \& Springel 2005).

\section{Results}

\subsection{Cosmological parameters}

We combined the linear dark matter power spectrum as inferred by Viel, Haehnelt \& Springel (2004) with the CMB data of WMAP (Spergel et al. 2003). The results in terms of cosmological parameters are summarised here: $\sigma_{8}=0.94 \pm 0.08 ; n_{s}=0.99 \pm 0.03$ (for models with no running, $1 \sigma$ error bars); $n_{s}=0.959 \pm 0.036$; and $n_{\text {run }}=-0.033 \pm 0.025$ (for a model that includes running). The data is thus consistent with a scale-free power spectrum and no running of the spectral index.

For slow-roll inflationary models we get the following constraints: $\epsilon_{V}=0.032 \pm 0.018$, $\eta_{V}=0.020 \pm 0.025$, and $\xi_{V}=0.015 \pm 0.014$. The constraint on the tensor to scalar ratio is $r=0.499 \pm 0.296$ (see Viel, Weller \& Haehnelt (2004) for the relation between these parameters and the spectral index and its running). The results above are in agreement with those of the SDSS collaboration (Seljak et al. 2004).

\subsection{Warm dark matter models}

Candidates of dark matter particles are generally classified according to their velocity dispersion which defines a free-streaming length. On scales smaller than the free-streaming length, fluctuations in the dark matter density are erased and gravitational clustering is suppressed. The velocity dispersion of Cold Dark Matter (CDM) particles is by definition so small that the corresponding free-streaming length is irrelevant for cosmological structure formation. That of Hot Dark Matter, e.g. light neutrinos (see the following section), is only one or two orders of magnitude smaller than the speed of light, and smooths out fluctuations in the total matter density even on galaxy cluster scales, which leads to strong bounds on their mass and density. Between these two limits, there exists an intermediate range of dark matter candidates generically called Warm Dark Matter (WDM). Viel et al. (2005) have explored two different scenarios: pure warm dark matter models that differ from $\Lambda$ CDM models by a suppression of power below a scale $\alpha$ (Fig. 2); and mixed models with CDM and gravitinos (or any warm dark matter particles that decouples with a $g_{*} \sim 100$, shown in Fig. 3).

We obtain a lower limit (in the case of pure warm dark matter models) of $m_{w d m}>$ $550 \mathrm{eV}(2 \sigma)$ for early decoupled thermal relics and $m_{w d m}>2.0 \mathrm{keV}(2 \sigma)$ for sterile neutrinos (that have never been in thermal equilibrium). In the case in which the gravitino density is proportional to its mass we find an upper limit $m_{\text {grav }}<16 \mathrm{eV}(2 \sigma)$. This translates into a bound on the scale of supersymmetry breaking, $\Lambda_{\text {susy }}<260 \mathrm{TeV}$, for models of supersymmetric gauge mediation in which the gravitino is the lightest supersymmetric particle (Viel et al. 2005). 

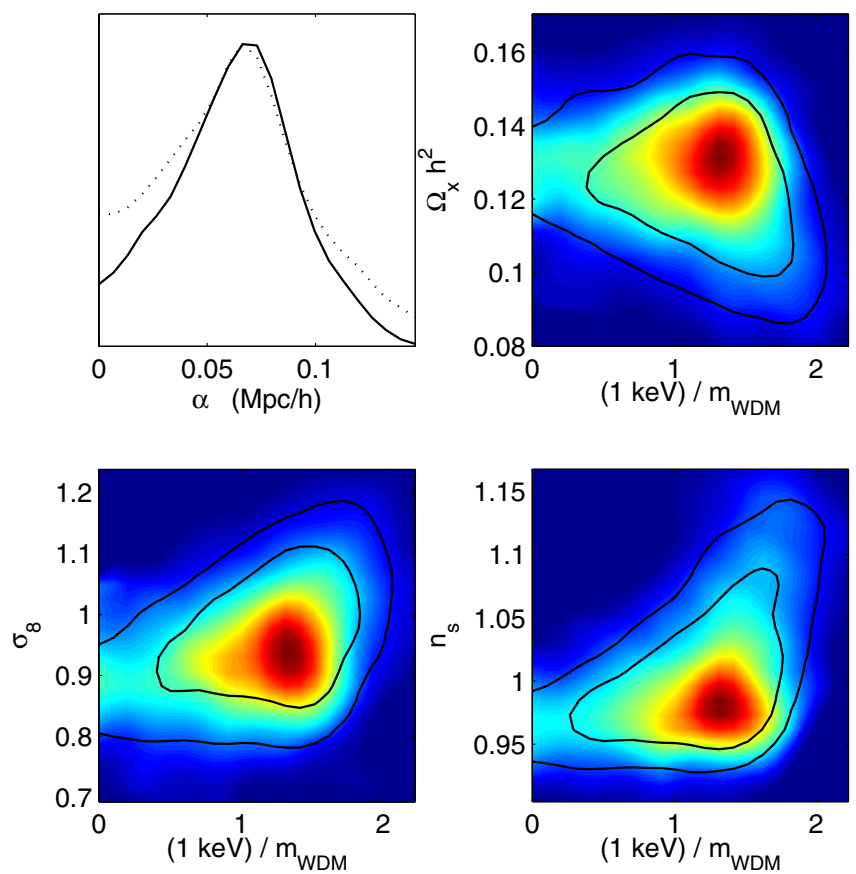

Figure 2. The scale of breaking for pure WDM thermal models. Power is suppressed below the scale $\alpha(\mathrm{Mpc} / \mathrm{h})$ and the suppression depends on the mass of the WDM particle. The line shows 1D-likelihood (upper-left panel, the marginalised result is shown as a continuous line); 2 D-likelihood ( $1 \sigma$ and $2 \sigma$ contours) in colours (the lines represent the marginalised results while the coloured contours are for the mean likelihoods) for $\Omega_{W D M}, \sigma_{8}$, and $n_{s}$ vs. the inverse of the WDM particle mass.

The values we get for the parameter $\alpha$ (which is related to the scale of free streaming) and $f_{x}=\Omega_{\text {grav }} / \Omega_{D M}$ are the following: $\alpha(\mathrm{Mpc} / h)=0.06 \pm 0.03$ and $f_{x}=0.05 \pm 0.04$ ( $1 \sigma$ error bars). Thereby, the concordance $\Lambda$ CDM model is perfectly consistent with the data.

\subsection{Neutrino masses}

We have explored constraints on the quantity $\Sigma m_{\nu}(\mathrm{eV})$, which is the sum of the mass of the light neutrino species. Both the CMB and large-scale-structure are important tracers of neutrino mass. At the time of decoupling neutrinos are still relativistic but become non-relativistic later in the evolution of the Universe if their mass is sufficiently high. As for WDM, but at larger scales, neutrinos free-stream out of their potential wells, erasing perturbations on smaller scales, producing a suppression of the amplitude, and leaving a characteristic feature at the transition scale. In Fig. 4 we plot 2D likelihood contours for several cosmological parameters. The lines indicate the same likelihoods when marginalised over the full set of parameters while the coloured contours are the mean likelihoods. The results shown here have been obtained by using the WMAP data set, the $2 \mathrm{dF}$ galaxy power spectrum (Percival et al. 2001) and the Lyman- $\alpha$ constraints of Viel, Haehnelt \& Springel (2004). We obtain the following values for the marginalised likelihoods: $\sigma_{8}=0.84 \pm 0.08, n_{s}=0.98 \pm 0.03, \tau=0.15 \pm 0.06, \Omega_{m}=0.33 \pm 0.06$, and $\Sigma m_{\nu}(\mathrm{eV})=0.33 \pm 0.27$ ( $1 \sigma$ error bars and for models which do not consider the running of the spectral index nor tensor contributions). 

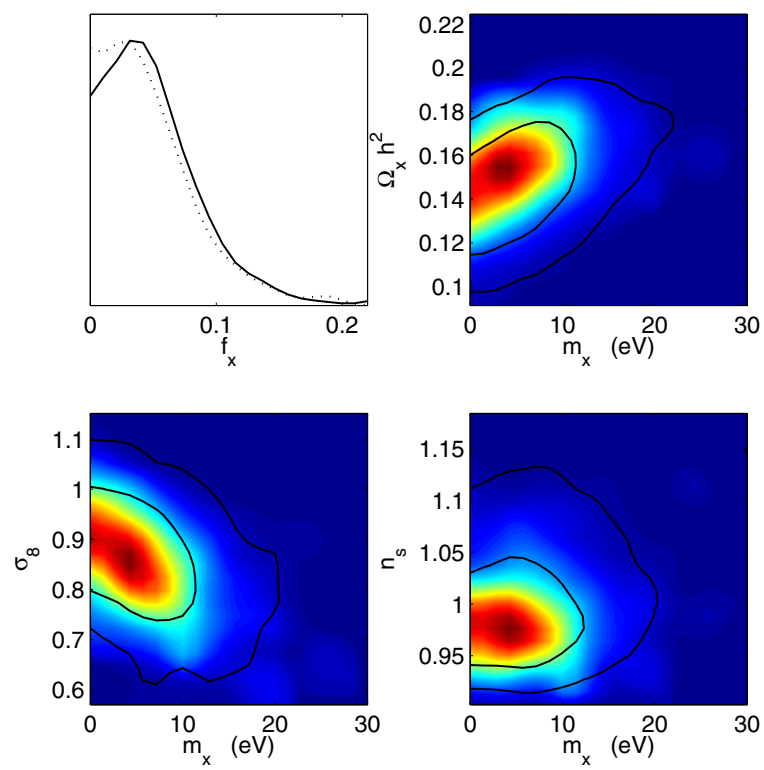

Figure 3. Contribution of a light gravitino to the dark matter density $f_{X}=\Omega_{\text {grav }} / \Omega_{D M}$ (1D-likelihood, upper left panel). The plots are the same as in Fig. 2 but for a model of mixed cold+warm dark matter. Contour plots for the cosmological parameters are now plotted vs. the gravitino mass (or any particles that decouples with a $g_{*}=100$ ).

\section{Conclusions}

The Lyman- $\alpha$ forest, the absorption by neutral hydrogen seen in the spectra of background QSOs, is a powerful laboratory for cosmology. We have used two samples of high resolution high signal-to-noise QSO spectra to infer the linear dark matter power spectrum at $z>2$ and at scales not probed by any other observables. The present uncertainties in the inferred values of the cosmological parameters are mainly due to the systematic errors involved in the numerical modelling of the Lyman- $\alpha$ forest data with hydrodynamical simulations. We have explored the differences between full hydrodynamical simulations and an approximate scheme based on the HPM approximation.

We have then combined the small-scale estimates of the Lyman- $\alpha$ forest with largerscale data sets such as the $\mathrm{CMB}$ and the $2 \mathrm{dF}$ galaxy power spectrum. The error bars on cosmological parameters that can be inferred from the combined analysis of CMB and Lyman- $\alpha$ forest data improve by a factor two those obtained by the CMB data alone. The final results point to a value of $\sigma_{8}=0.94 \pm 0.08$ and a spectral index $n_{s}=0.99 \pm 0.03$ ( $1 \sigma$ error bars) and no evidence for a running of the spectral index. These results are consistent with those obtained by the SDSS collaboration (Seljak et al. 2004). We have set constraints on the mass of a warm (thermal) dark matter particle to be $m_{w d m}>550 \mathrm{eV}$ and on the light gravitino to be $m_{\text {grav }}<16 \mathrm{eV}(2 \sigma$ error bars). Further constraints on the sum of the three neutrinos can be obtained and we get: $\Sigma m_{\nu}(\mathrm{eV})=0.33 \pm 0.27(1 \sigma$ error bar) by combining WMAP, $2 \mathrm{dF}$, and Lyman- $\alpha$ data.

\section{Acknowledgements}

I thank my collaborators: Julien Lesgourgues, Martin Haehnelt, Sabino Matarrese, Antonio Riotto, Volker Springel, and Jochen Weller. I thank the IAU for the award of a grant. The simulations were run on the COSMOS supercomputer at the Department of Applied Mathematics and Theoretical Physics in Cambridge. 

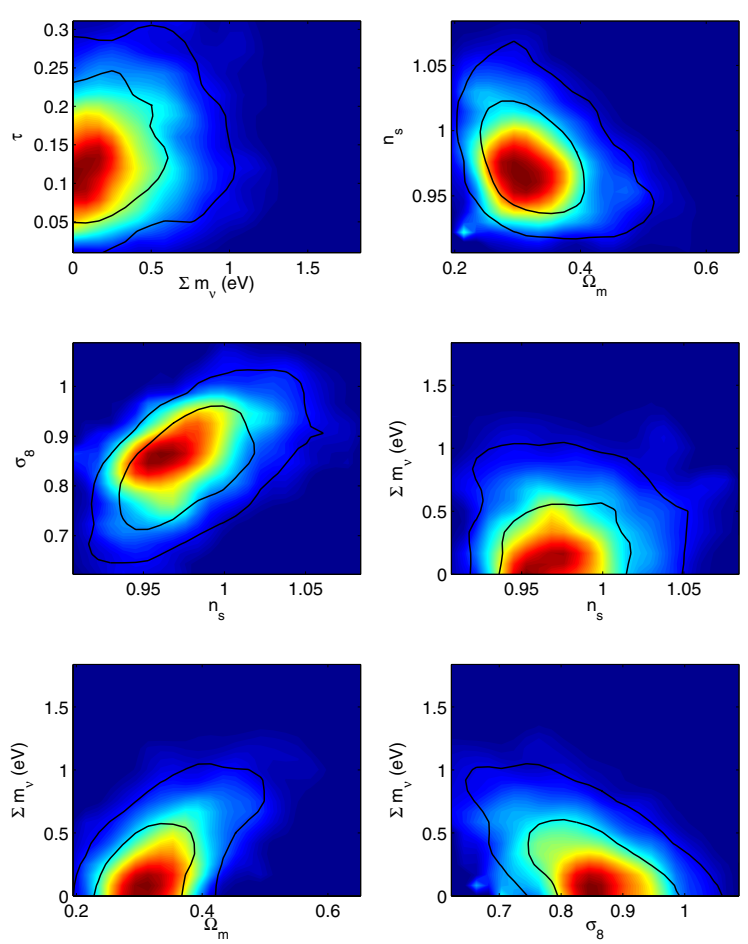

Figure 4. Constraints on the neutrino masses and on several cosmological parameters obtained by considering WMAP $+2 \mathrm{dF}+$ Lyman $-\alpha$. Mean likelihoods and marginalised ones are represented by contours and lines respectively.

\section{References}

Croft, R. A. C., Weinberg, D. H., Bolte, M., Burles, S., Hernquist, L., Katz, N., Kirkman, D., Tytler, D., 2002, ApJ, 581, 20

Croft, R. A. C., Weinberg, D. H., Katz, N., Hernquist L., 1998, ApJ, 495, 44

Gnedin, N. Y., Hamilton, A. J. S., 2002, MNRAS, 334, 107

Gnedin, N. Y., Hui, L., 1998, MNRAS, 296, 44

Hui, L., Burles, S., Seljak, U., Rutledge, R. E., Magnier, E., Tytler, D., 2001, ApJ, 552, 15

Kim, T.-S., Viel, M., Haehnelt, M. G., Carswell, R. F., Cristiani, S., 2004, MNRAS, 347, 355 K04

Lewis, A., Bridle, S., 2002, Phys. Rev. D, 66, 103511

McDonald, P., Miralda-Escudé, J., Rauch, M., Sargent, W. L. W., Barlow, T. A., Cen, R., Ostriker, J. P., 2000, ApJ, 543, 1

McDonald, P., et al., 2004, submitted to ApJ, astro-ph/0405013

Percival, et al., 2001, MNRAS, 327, 1297

Rauch, M., 1998, ARA\&A, 36, 267

Seljak, U., et al., 2004, astro-ph/0407372

Spergel, D. N., et al., 2003, ApJS, 148, 175

Springel, V., Yoshida, N., White, S. D. M., 2001, New Ast., 6, 79

Viel, M., Haehnelt, M. G., Springel, V., 2004, MNRAS, 354, 684

Viel, M., Haehnelt, M. G., Springel, V., 2005, astro-ph/0504641

Viel, M., Lesgourgues, J., Haehnelt, M. G., Matarrese, S., Riotto, A., 2005, Phys. Rev. D, 71, 063534

Viel, M., Weller, J., Haehnelt, M. G., 2004, MNRAS, 355, 23P

Viel, et al., 2004, proceedings of the XXXIXth Rencontres de Moriond on "Exploring the Universe", La Thuile, Italy, astro-ph/0405584 